\title{
Impacto de las TIC en la enseñanza - aprendizaje de las Ciencias Experimentales en el Bachillerato
}

\section{ICT Impact on Experimental Sciences teaching-learning at High School}

\author{
Darío Calderón Suárez ${ }^{1}$, Richard Torres $\operatorname{Vargas}^{2}$ y Manuel Segobia Ocaña ${ }^{2}$ \\ ${ }^{1}$ Unidad Educativa Ventanas \\ ${ }^{2}$ Universidad Técnica de Babahoyo \\ *decs101@hotmail.com
}

DOI: https://doi.org/10.26871/killkana_tecnica.v3i2.532

\begin{abstract}
Resumen
El trabajo de investigación aportó de forma significativa al mejoramiento de los métodos y técnicas de enseñanza aprendizaje de las Ciencias Experimentales; basándose en el empleo de las TIC como mecanismos de adquisición de aprendizajes significativos y así fortalecer los procesos didácticos empleados por los docentes de Bachillerato. La importancia radicó fundamentalmente en el desarrollo de competencias experimentales y tecnológicas de los estudiantes para la vinculación efectiva de este tipo de herramientas con la adquisición de sus conocimientos. Se emplearon métodos como el bibliográfico; el cual permitió conocer qué se ha investigado respecto al tema y cuáles fueron los resultados que se obtuvieron, y establecer el punto de partida inicial. Se emplearon métodos como el inductivo y deductivo; así como los métodos experimentales y hermenéuticos que de forma especial permitieron realizar el trabajo de campo para establecer las conclusiones y así medir el impacto que las TIC tienen en este campo de la enseñanza - aprendizaje. Dentro de los resultados obtenidos se evidenció que el $77 \%$ de los docentes que empleaban herramientas tecnológicas en sus procesos experimentales con sus estudiantes, lograban que ellos aprendieran de forma más dinámica en relación a las clases impartidas por los docentes que no las empleaban. En cuanto al aprendizaje se evidenció que el $95 \%$ de los estudiantes generaron aprendizajes significativos a partir del constructivismo. Esta investigación permitió que a partir de ello se incorporen con mayor compromiso el uso de las TIC en los procesos educativos.
\end{abstract}

Palabras clave: TIC, Ciencias Experimentales, Proceso enseñanza - aprendizaje, Constructivismo, Aprendizaje Significativo, Competencias.

\begin{abstract}
This research work has significantly contributed to the improvement of the teaching-learning methods and techniques of experimental sciences. The study is based on the use of ICTs as a significant learning acquisition mechanism aimed to improve the teaching processes used by secondary school teachers. The importance is mainly focused on the student's development of experimental and technological abilities and skills, to effectively link the use of these tools to their knowledge acquisition. The bibliographic method used in this research allowed us to know about previous research on this topic and the results obtained, as well as to establish the starting point. Additionally, other methods such as de inductive-deductive, and the experimental and hermeneutic ones have been used. They allowed us to carry out the research to establish the conclusions, and therefore measure the impact of ICTs in the educational field. The results obtained have shown that 77\% of the teachers who used technological tools in the experimental processes achieved more dynamic learning of their students, compared to the classes taught by the teachers who did not use these tools. In terms of learning, the results have shown that $95 \%$ of the students generated significant learning from constructivism. This research has made it possible for a greater commitment use of ICTs in the educational processes.
\end{abstract}

Key words: ICT, Experimental sciences, Teaching-learning process, Constructivism, Significant learning, Competences.

\section{INTRODUCCIÓN}

La enseñanza de las Ciencias Experimentales siempre ha significado un gran desafío dentro del campo de la educación, ya que es a través de ella que se pueden sistematizar aprendizajes significativos y por ende el descubrimiento de nuevos acontecimientos que marcan o delimitan la aprehensión de contenidos, con el significado que se requiere dentro de la formación del bachillerato. 
En los últimos tiempos es evidente que la educación del país ha tenido cambios significativos a nivel curricular. Se han introducido cambios que han beneficiado en cierta medida la enseñanza de las Ciencias Experimentales lo cual ha generado en los estudiantes la activación de los conocimientos previos, pero el problema radica en que muchos de los docentes que enseñan estas ciencias no se encuentran familiarizados con el empleo de la tecnología para potencializar estos procesos de interaprendizaje dinámico. De esta forma surge la interrogante entre el impacto que tienen las Tecnologías dela Información yla Comunicación(TIC) en la enseñanza - aprendizaje de dichas áreas.

Es importante destacar que el presente trabajo investigativo se desarrolló desde una perspectiva práctica, donde se pudieron emplear métodos como el inductivo y deductivo para conocer los casos particulares relacionados a las TIC y la enseñanza de las Ciencias Experimentales, así como el método hermenéutico empleado para la interpretación de los resultados y tener una visión panorámica de los resultados obtenidos, que permitirá establecer así una relación directa entre los procesos áulicos y las teorías que sustentan los aprendizajes significativos.

\section{DESARROLlO}

\section{A. Las teorías del aprendizaje y las TIC: Un factor clave en el aprendizaje de las Ciencias Experimentales}

Desde el punto de vista científico y sobre todo desde el punto de vista académico, las ideas previas que tiene consigo el estudiante permiten que construya su conocimiento a partir de lo que ya conoce; según lo propuesto por Gagné (1970) cuando manifiesta que "la información llega al sistema nervioso a través de los receptores sensoriales, para posteriormente procesarse y almacenarse en la memoria hasta que sea necesaria su recuperación. Si dicha información se corresponde con alguna previa puede pasar fácilmente a almacenarse, pero en caso contrario será necesaria la práctica y repetición del aprendizaje" [1]

Es así que cuando el docente trata de enseñar Ciencias Experimentales requiere conocer lo que el alumno sabe desde su cotidianidad. En este sentido el factor preponderante para que se pueda activar en forma eficiente es emplear procesos de motivación a la hora de recuperar la información, debe suceder alguna situación o estímulo que exija utilizar el aprendizaje almacenado, el cual ante dicho estímulo pasa a un hipotético generador de respuestas interno. Tras su paso por este generador se produce la conducta, teniendo en cuenta a la hora de escoger cuál aplicar el nivel de control y las expectativas propias y ajenas respecto a la conducta y la meta u objetivo a cumplir con ella.[1]

Así, la motivación actúa como motor del aprendizaje y a la vez hace que se creen más situaciones para poner en práctica lo aprendido, ya que crea más oportunidades en las que se detecta una situación en la que las nuevas habilidades adquiridas pueden ser útiles. [2]
En este sentido J. Bruner (1960) considera que los estudiantes deben aprender por medio del descubrimiento guiado que tiene lugar durante una exploración motivada por la curiosidad. Así, desde el punto de vista del aprendizaje por descubrimiento, en lugar de explicar el problema, de dar el contenido acabado, el profesor debe proporcionar el material adecuado y estimular a los aprendientes para que, mediante la observación, la comparación, el análisis de semejanzas y diferencias, etc., lleguen a descubrir cómo funciona algo de un modo activo. Este material que proporciona el profesor constituye lo que J. Bruner denomina el andamiaje. [3]

El uso de la tecnología mediante o las herramientas TIC dentro del aula, permiten que se generen aprendizajes significativos en los estudiantes que despierta además en ellos, el espíritu de investigación y el deseo constante de descubrir las ciencias, todo ello bajo el enfoque propuesto por D. Ausubel (1976); quien preconiza la enseñanza expositiva o el aprendizaje por recepción como el método más adecuado para el desarrollo del aprendizaje significativo. Esto facilita la parte experimental y la aplicación de herramientas esenciales para que el aprendizaje sea significativo. [4]

Para continuar progresando en el uso de las TIC en el ámbito de la educación, se hace necesario conocer la actividad que se desarrolla a nivel mundial, así como los diversos planteamientos pedagógicos y estratégicos que se siguen. La popularización de las TIC en el ámbito educativo comporta y comportará en los próximos años,una gran revolución que contribuirá a la innovación del sistema educativo, e implicará retos de renovación y mejora de los procesos de enseñanza-aprendizaje. [5]

Consecuente con esta mirada, Coll (2009: 117) conceptualiza a las tecnologías como "instrumentos psicológicos", en el entendido de que son "herramientas para pensar, sentir y actuar solos y con otros". De acuerdo con este autor, el hecho de concebir a las tecnologías como instrumentos psicológicos se apoya en su naturaleza simbólica, es decir, las sus posibilidades que ofrecen para representar, procesar, transmitir, compartir información, etc. Esta capacidad mediadora de las tecnologías, si bien es una potencialidad intrínseca, se hace o no efectiva, y en distintos grados y formas, según los contextos particulares, históricos y situados en donde se utilizan. En el presente caso, se considera que las aulas de ciencias son contextos educativos particulares en los que pueden, o no, hacerse realidad las posibilidades y potencialidades de las herramientas tecnológicas. [6]

La taxonomía cognitiva de James Bloom, se basa en la idea de que las operaciones cognitivas pueden clasificarse en seis niveles de complejidad creciente y dependiente; esto implica que si un alumno desea alcanzar un nivel superior, será necesario que primero domine los niveles inferiores que le preceden. Een este sentido, al comprender este proceso de aprendizaje se le facilitará al docente el empleo de las TIC en los procesos de enseñanza - aprendizaje de las Ciencias Experimentales. 


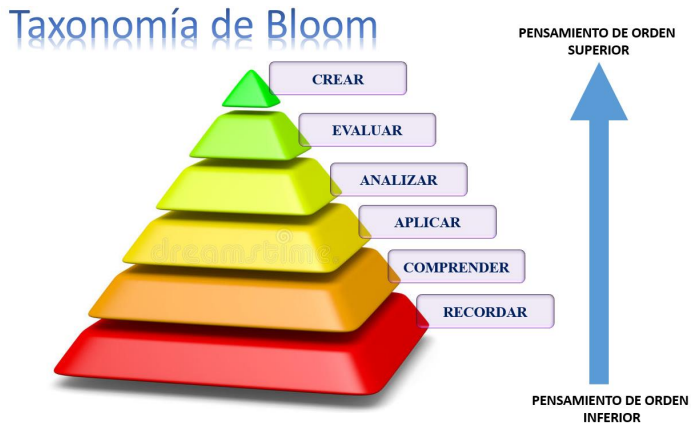

Fig. 1: Taxonomía de Bloom

Fuente: Adaptación de los autores

\section{MATERIALES Y MÉTODOS}

El estudio fue llevado a cabo en el cantón Ventanas, con una muestra de 233 docentes que imparten la Ciencias Experimentales en el Distrito 12D04 Quinsaloma - Ventanas quienes emplean o no las TIC en el proceso de enseñanzaaprendizaje.

Para dicho efecto se diseñó un cuestionario, el mismo que fue aplicado a todos los docentes que constituían la muestra de esta investigación. En este sentido se determinó la utilización de los métodos inductivo y deductivo. A partir de ellos se pudieron analizar los casos particulares y generales del empleo de las TIC. Se analizaron varias planificaciones y estrategias que planificaron los docentes.

Además se pudieron realizar varias observaciones áulicas en las que se evidenció el proceso de enseñanza - aprendizaje empleado por los docentes. Es importante destacar que la población que se empleó pertenece a las zonas urbanas y rurales del cantón, y abarca la totalidad de instituciones educativas.

\section{REsultados}

Históricamente, el avance y desarrollo tecnológico busca ser aprovechado por los sistemas educativos con el fin de convertir la educación que ofrecen en una educación de calidad. Desde la entrada de este siglo, la incorporación de las Tecnologías de la Información y Comunicación (TIC) en la educación se ha ofrecido como la piedra Filosofal capaz de mejorar y transformar los procesos y prácticas escolares. Esta incorporación se considera como indispensable en el contexto de un mundo cada vez más globalizado. [7]

Luego de haber obtenido los resultados de las encuesta a los 233 docentes que formaron parte de la presente investigación, se pudieron determinar los siguientes resultados.

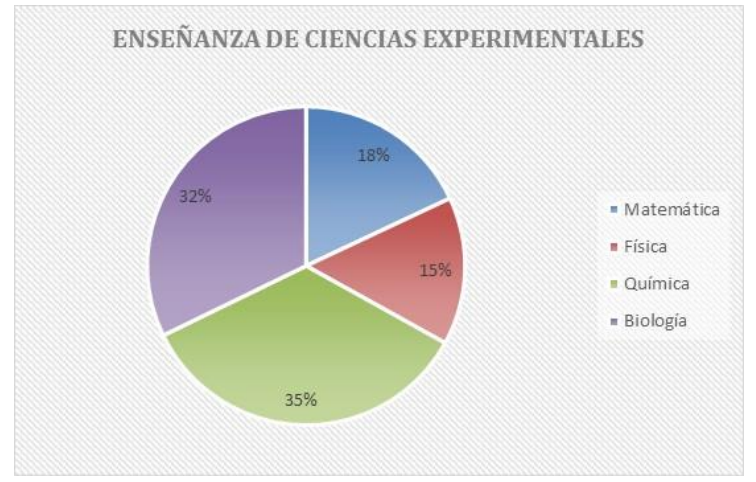

Fig. 2: Ciencias Experimentales que se imparten en la secundaria

Fuente: Encuesta a docentes de Ciencias Experimentales del cantón Ventanas

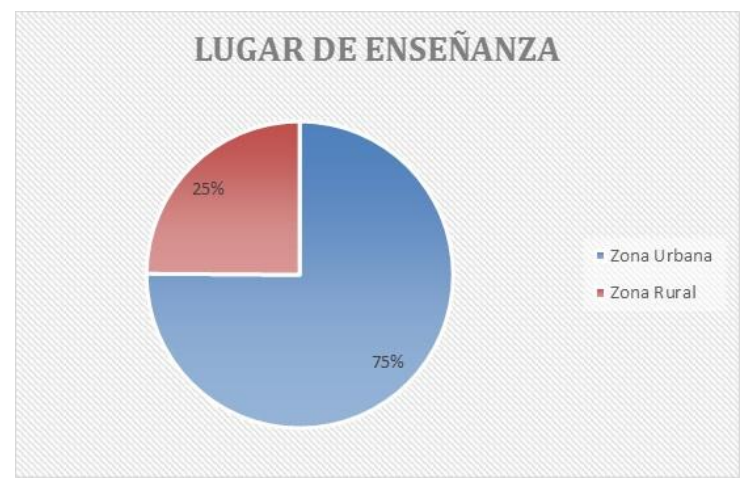

Fig. 3: Lugar de enseñanza de las Ciencias Experimentales Fuente: Encuesta a docentes de Ciencias Experimentales del cantón Ventanas

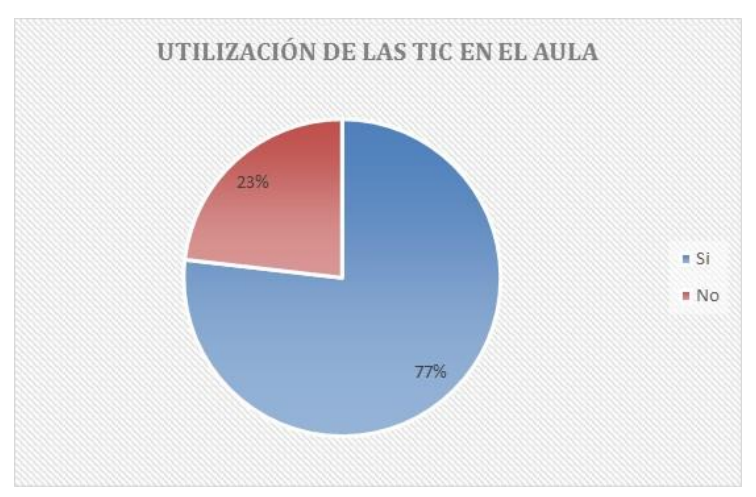

Fig. 4: Utilización de las TIC en el aula

Fuente: Encuesta a docentes de Ciencias Experimentales del cantón Ventanas 


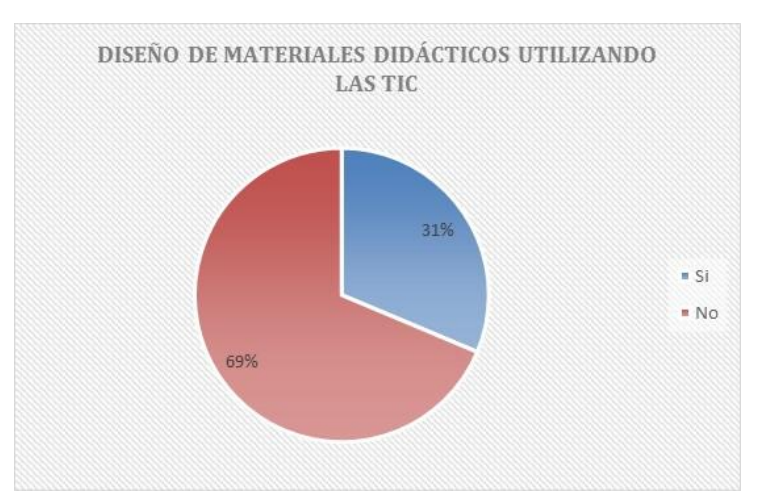

Fig. 5: Diseño de materiales didácticos utilizando las TIC Fuente: Encuesta a docentes de Ciencias Experimentales del cantón Ventanas

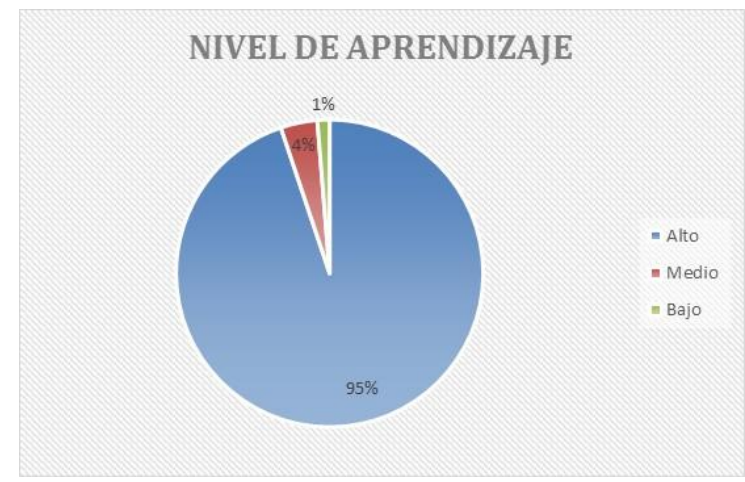

Fig. 6: Nivel de aprendizaje de los estudiantes

Fuente: Encuesta a docentes de Ciencias Experimentales del cantón Ventanas

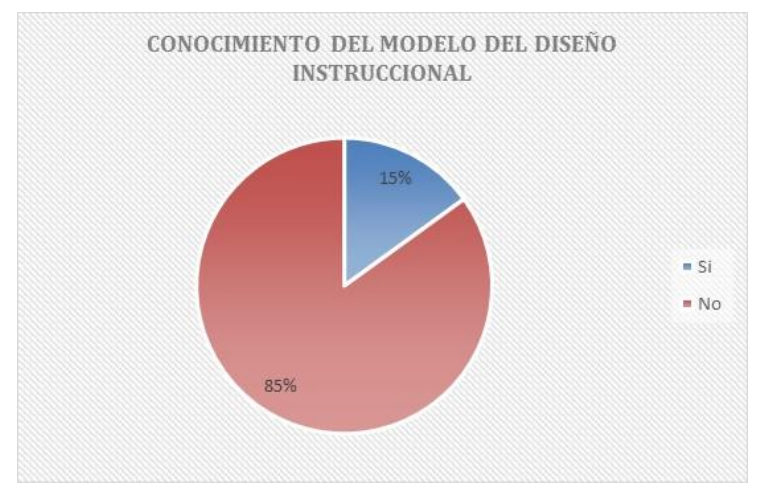

Fig. 7: Fundamento del Modelo de Diseño Instruccional Fuente: Encuesta a docentes de Ciencias Experimentales del cantón Ventanas

\section{Discusión DE RESUltados}

Según la UNESCO, las Tecnologías de la Información y la Comunicación (TIC) tienen un rol fundamental en el acceso universal a la educación, la igualdad en la instrucción, la enseñanza y el aprendizaje de calidad, la formación de docentes, y la gestión, dirección y administración más eficientes del sistema educativo. [8]
Por su parte, la UNESCO desarrolla un intenso trabajo en el ámbito del desarrollo de políticas y actividades que faciliten estos usos de las TIC. La alfabetización mediática e informacional de docentes, el aprendizaje móvil y electrónico, así como los sistemas de información sobre la administración de la Educación son algunas de las prioridades actuales. [8]

Se evidenció que un $35 \%$ de los docentes enseñan Química, facilita la utilización de las herramientas TIC dentro de los procesos de enseñanza - aprendizaje; mientras que un $32 \%$ se destacan en la enseñanza de la Biología, un $18 \%$ se centran en la enseñanza de las Matemática y un $15 \%$ de ellos son docentes de Física. Esto evidencia que las Ciencias Naturales tienen una mayor aplicabilidad de las Ciencias Experimentales dentro de las instituciones educativas.

Luego de obtener los resultados del estudio de campo, se apreció que el $75 \%$ de los docentes pertenecen a la zona urbana, en la cual ellos tienen mayores facilidades para emplear los recursos tecnológicos en la enseñanza de las Ciencias Experimentales, pero a pesar de ello muchos no las emplean. El $25 \%$ pertenecen a la zona rural; a pesar que presentan limitaciones en el sentido tecnológico, buscan la manera de acomodar la tecnología disponible para enfocarse en el cumplimiento de los objetivos.

Muy independiente de la zona donde se enseñen las Ciencias Experimentales (Biología, Química, Física y Matemática) se evidencia que un $77 \%$ de los docentes emplean estos recursos para generar aprendizajes significativos como lo propone Ausubel en su teoría del aprendizaje significativo. [4] Mientras que un $23 \%$ de ellos permanece indiferente a estos procesos innovadores para la enseñanza de las asignaturas que imparten.

Aunque el uso de las tecnologías dentro del aula muchas de las veces requiere la utilización de internet, se puede apreciar que el $69 \%$ de los docentes de Ciencias Experimentales no elaboran sus propios materiales, sino que buscan estos en la web acomodándolos a las necesidades educativas de sus estudiantes. El $31 \%$ de profesores elabora su material y emplean tecnología de punta o básica a fin de que los objetivos propuestos sean alcanzados por los estudiantes.

Es importante destacar que los docentes de las Ciencias Experimentales emplean recursos propios o acondicionados para poder emplear las TIC en sus procesos de enseñanza-aprendizaje. Al abordar esta pregunta, se pudo obtener como resultado que el $95 \%$ de los estudiantes mejoran su rendimiento académico cuando aprenden en forma visual o son partícipes de la construcción de su conocimiento mediante el empleo de las tecnologías de la información y la comunicación, lo que demuestra un relativo $1 \%$ de estudiantes que presentan un bajo rendimiento.

Al momento de indagar a docentes sobre innovaciones didácticas dentro del aula, como lo es en este caso el Modelo de Diseño Instriccional (Arshavskiy, 2014) [9]. Ellos supieron manifestar en un $85 \%$ que no conocen estos 
procesos de planificación que mejoran de modo significativo los aprendizajes de los estudiantes, de manera especial en las Ciencias Experimentales mediante el empleo de modelos específicos.

\section{CONCLUSIÓN}

Es importante destacar que en este trabajo se ha descrito en qué medida los docentes emplean las TIC en los procesos de enseñanza - aprendizaje de las Ciencias Experimentales, aunque muchos de ellos conocen cómo emplearlas presentan poca importancia a su utilización en el aula para generar aprendizajes significativos y por descubrimiento partiendo de la curiosidad y de la motivación que tengan.

Según lo expuesto por (Saez y Tortosa, 2008; Webb, 2009), no es suficiente con poner a la disposición de profesores y alumnos herramientas tecnológicas que potencialmente son complejas y poderosas o, en su caso, propuestas didácticas innovadoras. Es necesario estudiar la forma en que profesores y alumnos usan las herramientas tecnológicas en el desarrollo real de los procesos que ocurren en el aula de ciencias, para con ello trazar las coordenadas de los caminos más factibles que permitan una certera y atinada incorporación de las tecnologías en la educación científica. Avanzar en esta línea se vuelve relevante dado el papel crítico que tienen las ciencias en la formación integral de las nuevas generaciones. [10]

Por lo tanto, es importante tener creatividad por parte de la planta docente al momento de emplear las tecnologías disponibles en la institución educativa. Es así que Davis y Scott (1975) definen la creatividad como un proceso mediante el cual, de un problema que se presenta en la mente, ya sea imaginado, meditado, visualizado o supuesto, se origina o se inventa una idea, concepto, noción o esquema donde están presentes líneas nuevas no convencionales. [11]

Torrance (1976) la define como el proceso de percibir problemas o lagunas de información, formular ideas e hipótesis, verificarlas, modificarlas y comunicar los resultados. [12]

De la misma manera que el autor anterior, De la Torre (1997) asegura que creatividad es tener ideas y comunicarlas, y al relacionarla con la educación afirma que es un valor social y no solo científico-psicológico, es una exigencia social al igual que la educación. La estimulación creativa se convierte así en un cometido educativo como valor cultural actual. [13]

Se hace necesario implementar nuevos modelos como es el Diseño Instruccional (DI), el mismo que consiste en un proceso sistémico para la creación de material educativo a ser usado en procesos de enseñanza - aprendizaje apoyados por tecnología. El DI se considera una ciencia porque tiene como punto de partida las teorías del aprendizaje, y es un arte porque el proceso de diseño requiere de mucha creatividad. Tiene como objetivo ofrecer experiencias de aprendizaje agradables que facilitan la comprensión y permanecen en la memoria por mucho tiempo. [9]
Existen tantos modelos de DI como diseñadores instruccionales. Se puede encontrar un sin fin de propuestas que no necesariamente son aplicables a la realidad de un proyecto, es por esta razón que se realiza una revisión únicamente de los modelos más importantes, aplicables y probados:

- Modelo ADDIE (Análisis, Diseño, Desarrollo, Implementación, Evaluación): El modelo ADDIE es un proceso de diseño instruccional iterativo, en donde los resultados de cada fase pueden conducir de regreso a cualquiera de las fases previas. [14] [15]

- Modelo de Dick y Carey: Desarrollaron un modelo para el diseño de sistemas instruccionales basado en la idea de que existe una relación entre los estímulos, (materiales didácticos) y la respuesta que se produce en un alumno, (el aprendizaje de los materiales).[16]

- Modelo ASSURE (Análisis, Sentar objetivos, Seleccionar medios y materiales, Utilizar los métodos y materiales, Requerir la participación del estudiante, Evaluar y revisar): Tiene como base los nueve eventos de la instrucción de Gagné (a. ganar la atención, b. informar los objetivos, c. estimular los conocimientos previos, d. presentar material nuevo, e. guiar el aprendizaje, propiciar el rendimiento individual, f. brindar retroalimentación, g. evaluar la eficacia del rendimiento, incrementar la retención). El objetivo del modelo ASSURE es guiar al diseñador en la selección y utilización óptima de materiales educativos que se ajusten a las necesidades de los estudiantes. [17]

- Modelo ISD. (Preparación, Presentación, Práctica, Desempeño): El modelo rápido de aprendizaje acelerado (ISD) creado por David Meier, es ideal para diseñadores que trabajan con plazos ajustados, presupuesto limitado y contenido en constante cambio. Se concentra en las actividades de aprendizaje, más que en la presentación del contenido. [18]

- Modelo de aproximación sucesiva (SAM): El modelo SAM es otra forma de desarrollar cursos de maneramuy agil, creado por Michael Allen. Se concentra en la colaboración, la eficiencia y la repetición. SAM trabaja mucho con prototipos, más que cualquier otro modelo, pues espera que se cometan errores en el diseño, que gracias a estos se puedan identificar en fases tempranas del mismo y corregir con el menor impacto en tiempo y dinero. [19]

Desde el momento que los docentes de las Ciencias Experimentales comiencen a generar nuevas estrategias basadas desde una perspectiva innovadora para el empleo de las TIC dentro de los procesos educativos. Se está alcanzando lo propuesto por el Ministerio de Educación en su enfoque para la enseñanza de este tipo de asignaturas. “... el estudio de esta asignatura contribuye a reflexionar sobre la relación de la ciencia y la tecnología con la sociedad, y a evaluar, desde un punto de vista crítico y analítico, las implicaciones éticas y sociales de la aplicación e influencia de los nuevos descubrimientos en este campo, en múltiples contextos”. 


\section{REFERENCIAS}

[1] R. M. Gagné, A. de la Orden Hoz, and A. G. Soler, "Las condiciones del aprendizaje," 1987.

[2] A. Meza, "Psicología del aprendizaje cognoscitivo. hallazgos empíricos en los enfoques de piaget y gagné," 1979.

[3] H. D. Brown et al., Principles of language learning and teaching, vol. 4. Longman New York, 2000.

[4] D. P. Ausubel, J. D. Novak, H. Hanesian, et al., Psicología educativa: un punto de vista cognoscitivo, vol. 3. Trillas México, 1976.

[5] ADÉS, "J. y lejoyeux, m.(2003): Las nuevas adicciones internet, juego, deporte, compras, trabajo, dinero," Barcelona, Kairós.

[6] C. Coll, "Aprender y enseñar con las tic: expectativas, realidad y potencialidades," Boletín de la institución libre de enseñanza, vol. 72, no. 1, pp. 7-40, 2008.

[7] R. Carneiro, J. C. Toscano, and T. Díaz, "Los desafíos de las tic para el cambio educativo," 2009.

[8] "Las TIC en la Educación | Organización de las Naciones Unidas para la Educación, la Ciencia y la Cultura," Jul 2016.

[9] M. Arshavskiy, "Diseño instruccional para el aprendizaje en línea," USA: Your ElearningWorld, 2014.

[10] M. Area, "Las tecnologías de la información y comunicación en el sistema escolar. una revisión de las líneas de investigación," 2004.

[11] E. P. Torrance, G. Davis, and J. Scott, "Test para evaluar las habilidades creativas," Estrategias para la creatividad, pp. 159-174, 1975.

[12] E. P. Torrance, Educación y capacidad creativa. Ed. Marova, 1977.
[13] S. d. 1. Torre, Creatividad y formación: identificación, diseño y evaluación. 1997.

[14] G. Jamwal, "Effective use of interactive learning modules in classroom study for computer science education," 2012.

[15] "Instructional System Design: The ADDIE Model - A Handbook for Practitioners," Aug 2015.

[16] "Recursos Tecnologicos: TIC," Feb 2020.

[17] R. Heinich, M. Molenda, and J. D. Russell, Instructional media and the new technologies of instruction. Macmillan, 1989.

[18] D. L. Schwartz, X. Lin, S. Brophy, and J. D. Bransford, "Toward the development of flexibly adaptive instructional designs," Instructional-design theories and models: A new paradigm of instructional theory, vol. 2, pp. 183-213, 1999.

[19] D. Zhang, "Interactive multimedia-based e-learning: A study of effectiveness," The American Journal of Distance Education, vol. 19, no. 3, pp. 149-162, 2005.

Recibido: 10 de febrero de 2019

Aceptado: 18 de julio de 2019 